\title{
Formación ciudadana en Colombia: una propuesta para la universidad desde la perspectiva teórica de Jürgen Habermas
}

\author{
Carlos Fernando Cortés Baquero ${ }^{1}$ \\ Jhan Carlos Ramírez
}

Recibido: 09 de marzo 2012

Aceptado: 17 de mayo 2012

La formación ciudadana es una de las principales problemáticas de la formación universitaria actual en Colombia. Las cambiantes condiciones del entorno social nacional e internacional suscitadas por fenómenos como la globalización, las nuevas tecnologías de la información y las comunicaciones, la disminución del papel del Estado, entre otros, exigen una movilización y una participación cada vez más activa de ciudadanos íntegros, críticos, reflexivos, responsables y partícipes en la formulación e implementación de políticas públicas. Ante esto, resulta imprescindible comprender que el ámbito de lo público no se ciñe de manera exclusiva al espacio «privilegiado» a la hora del ejercicio de las responsabilidades administrativas y la práctica de principios éticos (provenientes de entes de carácter público). Por el contrario, aquel ámbito debe manifestarse en un sentido amplio como el espacio público donde el ejercicio de la opinión pública y la discusión crítica cobren especial importancia en la construcción de políticas públicas. Estas últimas, dada su pretensión de legitimidad y validez, se obligan a la aprobación y al asentimiento ciudadano constituyendo así su principio y su fin.

No obstante, la aceptación dogmática de que la democracia y su ejercicio pleno se agotan en el voto, ofrece al individuo una vaga idea de las responsabilidades y prácticas que deben orientar la práctica ciudadana. Esto ya que no sólo distrae al individuo de sus responsabilidades, sino que niega cualquier intento de libertad y autonomía y con ello, la posibilidad de deliberar sobre los asuntos públicos.

1 Carlos Fernando Cortés Baquero Egresado de la Facultad de Derecho de la Universidad Militar Nueva Granada, con formación en el área de Ciencias Humanas, específicamente en el área del Derecho Administrativo General y Colombiano, con conocimientos en administración pública, gestión pública y organizaciones, con énfasis en diseño, mplementación y análisis de políticas pública. Investigador Universidad Militar Nueva Granada. 


\section{Carlos Fernando Cortés Baquero y Jhan Carlos Ramírez}

Formación ciudadana en Colombia: una propuesta para la universidad desde la perspectiva teórica de Jürgen Habermas, artículo de reflexión

De ahí que, partiendo de las perspectivas de formación ciudadana de John Rawls y Jürgen Habermas, convenga preguntarnos si uno de estos enfoques teóricos permite potenciar, fomentar y conducir la práctica ciudadana y el compromiso social en Colombia a través de la formación ciudadana que ofrecen las universidades y si estas propuestas pueden dar lugar a diversas alternativas que faciliten la promoción de los principios de libertad y autonomía.

Por consiguiente, y tomando como punto departida los postulados teóricos de Jürgen Habermas, nuestra propuesta aboga por la promoción de los principios de libertad y autonomía plena en la formación universitaria en Colombia . Desde esta perspectiva la exaltación de la opinión, la deliberación y la discusión crítica en las universidades son los únicos elementos posibilitadores y formadores de discursos de opinión y voluntad pública, toma de posturas y comunicación de contenidosesenciales en la promoción de la práctica ciudadana, la responsabilidad y el compromiso social en Colombia ${ }^{3}$.

\section{Condiciones de la Formación Ciudadana actual en Colombia}

Una de las características más sobresalientes de la formación ciudadana en Colombia, al igual que en muchos otros países de corte «occidental», descansa sobre los preceptos de ciudadanía, generalmente formulados alrededor de intereses parcialmente ajenos a la promoción de la ciudadanía misma así como en proyectos de nación impuestos (Mesa Arango, 2008). Esto como consecuencia de la acción dinámica del modelo neoliberal hegemónico presente en el orden mundial que se relaciona de manera directa con las orientaciones inherentes a una economía de mercado (Peralta Duque, 2007).

El Estado de hoy prioriza las relaciones de asociación y negociación con los sectores financieros dominantes por encima de su obligación de facilitar el desarrollo de una sociedad civil democrática, lo que conlleva a la delegación de competencias públicas en sectores no estatales. Igualmente, da lugar a una significativa y creciente injerencia de actores corporativos y organizacionales de carácter privado en asuntos públicos, constituyendo una tendencia mundial- de «achicamiento» que revela las más oscuras intenciones de los capitales transnacionales.

Con la Constitución de 1991, el Estado colombiano apostó a construir una ciudadanía que tenía como referente el modelo cívico, considerado el tipo ideal de moral pública, donde los valores fundamentales deberían ser «el respeto del orden jurídico, la responsabilidad de los funcionarios, la participación ciudadana y la protección de los derechos individuales» (Hurtado y Naranjo en Jiménez 
Martínez, s.f.: 14). Por su parte, Mesa Arango (2008) en su artículo «La formación ciudadana en Colombia», distingue algunos perfiles de la propuesta orientadora de formación ciudadana de las instituciones educativas en Colombia. En consonancia con el modelo cívico, y sin que exista consenso ni unanimidad al respecto en un país multicultural y plural como el nuestro, Mesa Arango aboga por la formación de «[c]iudadanos observadores de la Ley y el orden, buscadores y propiciadores de la paz, participativos, críticos de lo establecido, defensores de los derechos..., cumplidores de sus deberes» (2008: -).

Distintas instituciones educativas en Colombia han adelantado esfuerzos dirigidos a la implementación de proyectos de formación ciudadana al amparo de la Constitución Nacional de 1991 y la Ley $115^{4}$. Sin embargo, más allá del punto de vista estrictamente jurídico, el modelo cívico se distancia de manera flagrante de aquellas condiciones determinantes en la configuración de la realidad nacional del país al prescindir en sus consideraciones de aspectos sumamente relevantes como la escasa legitimidad del papel del Estado, los altos niveles de pobreza y de exclusión social, las distintas modalidades de la guerra, el narcotráfico, entre otros (Jiménez Martínez, s.f.: 14). En efecto, al desconocer la realidad política y social del país, el Estado se inclina de manera equívoca hacia la promoción de una ciudadanía cívica determinada por la definición de valores a priori, la reproducción irreflexiva de ideas y costumbres, y la proliferación de intereses y prácticas políticas ineficaces y corruptas. Ninguno de estos elementos corresponde a las prácticas ciudadanas que se suscitan en medio de un ambiente de guerra, pobreza y exclusión social.

Afirmar en el ámbito académico que la formación ciudadana en Colombia es un asunto político más que académico (Mesa Arango, 2008), resulta más que impreciso. Especialmente si se tiene en cuenta -en consonancia con Mesa- que los principios rectores orientadores de la formación ciudadana en Colombia, inmersos en el modelo cívico, han girado desde mediados del siglo XX en torno a la historia patria y los valores cristianos (cuyo propósito apunta al control del individuo aislado desde el poder central bajo el marco de valores católicos ). Al mismo tiempo, Mesa (2008) reconoce la incorporación de nuevos elementos que, aunados al discurso emergente del desarrollo, influyen en la formación ciudadana en Colombia. Este es el caso de los derechos humanos, la globalización, la democracia y de forma más reciente, la diversidad cultural; elementos impulsados por los gobiernos locales a partir de los cuales -se intentan construir modelos alternos al modelo neoliberal.

Los elementos precedentes nos permiten afirmar que la formación ciudadana en Colombia sufre de una perjudicial parálisis que desconoce, entre otros aspectos, 
su estrecho vínculo con el paso de la historia, la transformación permanente de «las prácticas y concepciones sobre el ser humano, la educación, la pedagogía y la política» (Jiménez Martínez, s.f.: 2) y la noción actual de política así como su ampliación a prácticamente la totalidad de los aspectos de la vida social humana. Desconoce, finalmente, el papel de la sociedad y su responsabilidad junto al Estado; esferas que consideradas en conjunto obligarían a superar el malformado concepto de formación ciudadana (como aquella que solo se ocupa de las relaciones de los ciudadanos con el Estado y con lo público).

En tanto, en Colombia la formación ciudadana continúa siendo un concepto al que no se le presta suficiente importancia al interior de la educación y por lo tanto no permite el ejercicio de la práctica social ciudadana, el compromiso y la responsabilidad social. Al carecer de fundamentación ética, filosófica y política de fondo, se evidencia una orientación hacia la manipulación velada de la participación política ciudadana de la sociedad colombiana (Jiménez Martínez, s.f.: 8). Resulta pues imprescindible comprender que la forma en que se desarrolla la cultura política en el entorno en donde actúan las instituciones educativas, incide indefectiblemente sobre el impacto que esta ejerce sobre el mismo (Peralta Duque, 2007: 166).

\section{Formación Ciudadana en Habermas}

Para Habermas, la democracia deliberativa es un concepto que obedece a prácticas que se entremezclan, se complementan y se convalidan en el ejercicio pleno de una democracia legítima. Es a través de este supuesto como Habermas intenta espigar un modelo de ciudadano propicio a los preceptos de «racionalidad comunicativa», participación, deliberación y práctica ciudadana.

\section{Democracia deliberativa}

Habermas redime del republicanismo el admirable propósito de vincular los miembros de la comunidad al ejercicio del poder, así como la posibilidad de llegar a acuerdos. Sin embargo, es aquí mismo donde Habermas reconoce su principal problema. Ante la imposibilidad del republicanismo de llegar a acuerdos en medio del pluralismo de culturas y formas de vida de la sociedad actual, «no puede haber acuerdo total sobre todo y es posible superar las divergencias sólo en los aspectos básicos de la convivencia, en un nivel de consenso ético, pero sobre cuestiones políticas opera la negociación y el compromiso» (Pallas, 2004: -). De tal modo, para Habermas estos últimos aspectos son solo posibles mediante la comunicación y, de manera más concreta, a través de la discusión y la argumentación (Velasco en Pallas, 2004). 
En esencia, Habermas propone, mediante el ejercicio de la racionalidad comunicativa como alternativa de entendimiento ciudadano en una democracia, la posibilidad de indicar a los actores del Estado qué deben hacer. Este recurso, «ofrece una guía [....] para la reconstrucción de aquel entrelazado de [....] discursos formadores de opinión, en el que está asentado el poder democrático ejercido de conformidad al Derecho» (Loos, s.f.: 242). Dicho de otra manera, se emplea como fórmula para obtener la satisfacción de legitimidad y validez del sistema en la medida en que los sujetos, como parte de una sociedad en constante interacción y mediante la comunicación, llevan a cabo sus correspondientes idealizaciones, imprimiéndole al resultado comunicativo una «fuerza vinculante». Dicha «fuerza vinculante» no es otra cosa que el resultado de convicciones motivadas de manera racional, confiriendo de esta manera al Derecho - a partir del proceso de entendimiento ciudadano- su pretensión de legitimidad y universalidad(y cuya fuerza integradora social en todo caso no puede fundarse única y exclusivamente en factores de decisión éticos de carácter institucional).

A través de la participación ciudadana, Habermas estima como válidas todas aquellas normas que permitan que «los que puedan verse afectados por ellas pudiesen prestar su asentimiento como participantes en discursos racionales» (Loos, s.f.: 242). Así pues, intenta demostrar que la soberanía popular cobra forma jurídica en la medida que es posible la formación discursiva de la opinión y la voluntad, y que esta se halla profundamente enraizada en principios morales, dando así lugar a la configuración de la autonomía política. De ahí que Habermas coloque en un mismo nivel la moral y el Derecho positivo e identifique la soberanía popular como intersubjetiva, pues ésta se encuentra condicionada a procedimientos que funcionan como «esclusa para la racionalización discursiva de las decisiones de una administración y un gobierno ligado al derecho y la ley» (Pallas, 2004: -).

El modelo de democracia deliberativa se sustenta a partir de las preferencias procesadas en la opinión pública, en donde tienen lugar la formulación y la construcción de programas y políticas públicas. Al ser implementadas, estas políticas otorgan fuerza al marco normativo y jurídico del Estado y le proveen elementos de estabilidad e identidad, haciendo a un lado todo lo que no pueda pasar por el proceso de deliberación pública. El núcleo de la propuesta habermasiana radica entonces en las condiciones comunicativas bajo las cuales «el proceso político tiene para sí la presunción de producir resultados racionales porque se lleva a cabo en toda su extensión de modo deliberativo» (Loos, s.f.: 243).

Habermas cree que es posible consensuar acerca de la legitimidad del procedimiento legislativo, y el ejercicio del poder soberano (Pallas, 2004). Para tal fin, los ciudadanos deben participar activamente del proceso democrático, desencadenando «las libertades comunicativas en la esfera pública política» (Pallas, 2004: -) para obtener de esta manera, la domesticación del poder ilegítimo y el empleo del poder administrativo en igual interés de todos (poder público 
derivado del poder comunicativo). Los resultados obtenidos con estas prácticas entran en juego con el poder potencial que ejercen otros actores capaces de hacer valer sus intereses, y por otra parte, con el poder administrativo que ejercen quienes ocupan cargos públicos.

Los compromisos, resultado de los distintos discursos y negociaciones, se implementan finalmente en «acuerdos en forma y lenguaje del Derecho» (Loos, s.f.: 244), transformándose así en poder administrativo. No obstante, es importante afirmar que Habermas en su teoría del discurso no le asigna legitimidad al Derecho y al ejercicio del poder político sobre la base del contenido jurídico, sino que este viene determinado por la acción del poder comunicativo, a través de cuyo acuerdo éste es ordenado. En ese sentido, el Estado se hace necesario e indispensable como «poder sancionatorio, organizativo y ejecutivo» (Loos, s.f.: 243). El modelo normativo de democracia deliberativa de Habermas, en últimas, tiene en cuenta el derecho de los ciudadanos a mantener su propia forma de vida cultural, pero también la obligación de aceptar «el marco político de convivencia definido por los principios constitucionales y los derechos humanos»(Habermas en Pallas, 2004: -), vinculando así lo particular frente a lo universal.

Resumiendo, para Jürgen Habermas el proceso de formación de la opinión y de la voluntad política en el espacio público está determinado, no sólo por la capacidad de autocomprensión ética, sino también por la fundamentación moral, el acuerdo de intereses y compromisos y la coherencia jurídica (Ortiz Leroux, : 57).

\section{Promoción de la práctica ciudadana y el compromiso social desde la Universidad}

Las consideraciones sobre las condiciones presentes en la formación ciudadana en Colombia -señaladas en párrafos anteriores- apuntan a la necesidad de orientar tal formación desde la universidad. Igualmente, indican la importancia de dirigirse hacia la comprensión de la educación social actual bajo las perspectivas de los diversos factores políticos que la explican y dan forma. Esto hace indispensable que la formación ciudadana en Colombia pase por el análisis, la reflexión, la crítica y la discusión de las determinaciones de la economía los sujetos que constituyen la ciudadanía, sus prácticas, las relaciones políticas entre las instituciones y los individuos, el conflicto interno del país las formas impuestas de política, entre otros aspectos (Jiménez Martínez, s.f.: 18-19).. Todo lo anterior como un intento por interpretar los cambios históricos de la colectividad y sus nuevas realidades sociales así como por aportar a la construcción de una vida ciudadana (con fundamentos 
en la autoreflexión, la discusión y el análisis) y al desarrollo de prácticas sociales innovadoras que permitan a la colectividad interpretar su presente y decidir por sí misma su propio futuro. Por esta razón la formación ciudadana en Colombia, considerada como saber dirigida a todos los sujetos sociales (político o ciudadano), exige potenciar desde la universidad su capacidad de participación, deliberación y acción frente al Estado y la sociedad civil (Jiménez Martínez, s.f.: 3).

Los elementos de la acción comunicativa de Jürgen Habermas entonces, constituyen una propuesta para la universidad al encaminarse en la promoción de la práctica ciudadana y el compromiso social mediante los procesos de formación de la voluntad y la opinión pública. Se aboga de ese modo por un determinado tipo de ciudadano que advierta la democracia como el resultado de un proceso de discusión y argumentación de la voluntad colectiva. y que comprenda que más allá de los intereses particulares e individuales existen intereses comunes, producto del obligado vínculo social. Esto da cabida, partiendo de condiciones específicas y concretas, a la configuración de ciudadanías activas, producto de aquellos procesos autoconscientes tanto individuales como colectivos (Jiménez Martínez, s.f.).

No obstante, es necesario adoptar esfuerzos cooperativos para lograr una práctica (participación ciudadana) que resulta imprecisa imponer: nadie puede ser obligado a ser ciudadano. Al respecto Habermas señala:

[H]oy la masa de la población sólo puede ejercer ya sus derechos de participación política por vía de integrarse en, y ejercer influencia sobre, la circulación informal de la opinión pública, circulación no organizable en conjunto (Pallas, 2004: -).

Así pues, se pueden esperar resultados racionales siempre y cuando las instancias parlamentarias y/o administrativas permanezcan sensibles a la formación informal de la opinión, la que no puede provenir sino de espacios públicos autónomos.La importancia de los espacios públicos formados de manera más o menos espontánea, pluralistas, informales, y y frágiles debido a los efectos de la represión y la exclusión, radica en que es allí donde se elaboran y surgen las propuestas políticas concretas de valor real. Esto supone una adecuada articulación de las necesidades a través del intercambio comunicativo, controlando y procesando la aplicación efectiva de las normas y principios constitucionales, y produciendo variedad de argumentos, influencias y opiniones.

De ahí que para Habermas el espacio de la formación de la opinión pública se constituya en «una red abierta e inclusiva de espacios públicos subculturalespluralistas - que se solapan unos con otros, con límites temporales, sociales y objetivos fluidos» (Pallas, 2004: -) que se forman de manera más o menos 


\section{Carlos Fernando Cortés Baqueroy Jhan Carlos Ramírez}

Formación ciudadana en Colombia: una propuesta para la universidad desde la perspectiva teórica de Jürgen Habermas,

espontánea, constituyéndose en un «complejo salvaje» como consecuencia de su confusa estructura. En consecuencia, la esfera pública en Habermas no es una organización institucional, sino que se describe mejor como

[...] una red para la comunicación de contenidos y tomas de postura, es decir, de opiniones, y en él los flujos de comunicación quedan filtrados y sintetizados de tal suerte que se condensan en opiniones públicas agavilladas en torno a temas específicos (Habermas en Pallas, 2004: -).

Esta esfera pública, además, tiene la ventaja de ser un medio de comunicación más propenso al autoentendimiento, producto de la intersubjetividad, por cuanto en ella los problemas que aquejan al conjunto de la sociedad se abordan, se discuten, tematizan y se concluyen.

Y es aquí justamente donde estriba la importancia de la universidad en Colombia, que al ser considerada como parte vital de esa red comunicativa, se constituye en un escenario autónomo privilegiado para la discusión y la reflexión acerca de lo público. Dada su capacidad para condensar diversidad de opiniones e intereses y llevar a cabo procesos dinámicos de comunicación, la universidad es relevante para el proceso desde su función de extensión, orientados al logro de acuerdos y consensos en virtud a la pluralidad, la informalidad y la espontaneidad que la asisten.

Nuestra propuesta, sin pretender recetar fórmulas de carácter estructural, pasa por una consideración socializadora de la educación de tipo constructivista. Se busca que desde la Universidad se promueva el desarrollo y la actividad mental del individuo, entendiendo que es una persona única, perteneciente a un contexto y a un grupo social determinado en el que puede influir, y en el que a su vez, está sujeto a influencia. Este modelo precisa que los docentes, consideren hacerse preguntas como ¿qué tipo de sujeto quiero formar?, ¿qué tipo de formación se requiere? y ¿para qué sociedad? (Torres de Torres, s.f.).

De acuerdo con este planteamiento, autores como Ausbel, Novak y Hanesion, piensan que el objetivo de la enseñanza es que

el estudiante se apropie del conocimiento de forma integral, dejando que el individuo actué de manera autónoma y con mas libertad de pensamiento, para llegar a alcanzar un conocimiento generador, que no se acumula sino que actúa, enriqueciendo la vida de las personas y ayudándolas a aprender del mundo y a desenvolverse en él (Torres de Torres, s.f.).

Lo anterior se ajusta a los principios rectores de la propuesta de Habermas, que busca promover los ideales de libertad, desarrollo y autonomía ciudadana, ampliando la capacidad de organización y expresión ciudadana. 


\section{John Rawls y la «autonomía política»}

En la teoría de democracia deliberativa anotábamos que Habermas concibe al Estado como un poder sancionatorio, organizativo y ejecutivo que sustenta el marco político de convivencia ciudadana (fundamentado en principios constitucionales y los derechos humanos). Para John Rawls, sin embargo, el objeto primario de la justicia es la estructura básica de la sociedad, es decir, el modo en que la constitución política distribuye los derechos y deberes fundamentales e influye sobre las perspectivas de vida del hombre. John Rawls, describe la sociedad como una asociación de personas más o menos autosuficientes, que dan lugar a un sistema de cooperación planificado para promover el bien de todos aquellos que toman parte de él. Es una asociación donde se reconoce en la cooperación la posibilidad de obtener un mejor modo de vida - en la medida en que cada individuo se valga de sus propios esfuerzos.

En efecto, John Rawls en su teoría de la justicia considera que los principios de justicia corresponden al objeto de un acuerdo original hipotétitco (que no se lleva a cabo de manera efectiva) entre personas racionales, libres e iguales en una situación contractual justa, con el objeto de promover sus propios fines en una situación inicial de igualdad. La imparcialidad de la situación contractual a la que Rawls denomina posición original, viene garantizada por el velo de la ignorancia, que sitúa a las personas en pie de igualdad frente a los demás e impide a los participantes del acuerdo poseer la totalidad de los conocimientos especializados; incluso los relacionados con su propia identidad y con la sociedad a la cual pertenecen (Caballero, 2006: 2). De tal modo, no se confiere a nadie desventajas ni ventajas, haciendo posible establecer consensos sobre lo justo entre los individuos involucrados.

Como señala Carvajal Correa (1999), la posición original de Rawls idealiza el supuesto de que los representantes de los ciudadanos, que se consideran a sí mismos libres e iguales en tanto individuos racionales, son suficientes para alcanzar los principios razonables de justicia que rigen el modelo de una sociedad democrática constitucional bien ordenada (donde los asuntos sustantivos son discutidos y acordados a priori por expertos, capaces de reconocer la realidad y forjar su propio destino, mediante el logro de consensos). A diferencia de Habermas, quien aboga por el ejercicio de la racionalidad comunicativa, Rawls afirma que la razón pública viene dada por el razonamiento de legisladores, jueces y representantes de la ciudadanía en general. Así, cuando se formulan asuntos constitucionales que constituyen la justicia básica, se da por sentado que las partes racionales pueden representar a cabalidad a los individuos que integran una sociedad, frente a la incapacidad que de ésta para alcanzar acuerdos racionales, mediante la negociación, y adoptar la perspectiva recíproca que debe exhibir la justicia. 
Asimismo, Rawls agrega que los ciudadanos libres e iguales representados por aquellos individuos racionales inmersos en condiciones razonables, «se contemplan como alcanzando por sí mismos un acuerdo acerca de dichos principios políticos en condiciones que representan a aquellos ciudadanos igualmente como razonables y racionales» (Carvajal Correa, 1999: -). De este modo, para Rawls, las libertades como resultado del proceso constituyente corresponden al fruto de las deliberaciones producidas a lo largo del tiempo. Por esta razón, al ser fijadas en la carta de derechos de la constitución, suponen el resultado manifiesto de la voluntad popular, de manera que no constituyen «una constricción externa sobre la soberanía popular» (Carvajal Correa, 1999: -). Consecuencia de esta interpretación, es el alcance de la autonomía política ciudadana, que expresa de manera estricta los intereses políticos de los ciudadanos con respecto a sus instituciones en un proceso continuo de construcción constitucional. Esto, si se tiene en cuenta que aún los ciudadanos de una sociedad bien ordenada están en capacidad para revisar y cambiar su constitución y sus leyes cuando las circunstancias lo exijan.

Por consiguiente, la autonomía política de los ciudadanos no puede darse por fuera de una constitución que «razonablemente justa...garantice su libertad e igualdad, con todas las leyes y preceptos subordinados adecuados que regulan la estructura básica» (Rawls, 2000: 77). Esto responde así a las objeciones centrales a su teoría, dirigidas a cuestionar el concepto de autonomía política, que en su versión refinada de la justicia como imparcialidad, cumple la función de mantener su estabilidad (Carvajal Correa, 1999). Rawls propone una teoría ideal, y por lo tanto normativa, de la justicia. De ahí, que su mérito, puramente procedimental, radique en la posibilidad de contar con «alguna noción de lo que es justo para, desde allí, evaluar la gravedad de las imperfecciones reales y establecer el mejor modo de acercarse a ese ideal» (Caballero, 2006: 21).

Por su parte, en Habermas política y comunicación forman un conjunto indisoluble en su modelo de sociedad democrática. De tal manera, si la política es esencialmente comunicación, entonces la fundamentación de un gobierno democrático no podrá agotarse exclusivamente en el voto «sino en consideraciones pragmáticas, discursos de autocomprensión y justicia» (Ortiz Leroux, 2006: 57). La política deliberativa de Habermas viene determinada por la interrelación entre la formación de la voluntad democráticamente constituida en espacios institucionales (públicos) y la construcción de la opinión informal en espacios extra-institucionales, cuya suma da lugar a la conformación de modelos administrativos democráticamente legítimos. Esto supone que el sistema político deba estar ligado entonces a las redes periféricas constituidas por la esfera pública a través de un flujo de redes de comunicación que «parte de redes informales de esa esfera.., se institucionaliza por medio de los cuerpos parlamentarios y toca el sistema político influyendo en las decisiones tomadas» (Feres en Ortiz Leroux, 2000: 58). 
La democracia en Habermas debe entenderse entonces como el resultado de un proceso racional de comunicación de contenidos, que dan lugar a la discusión y argumentación para la formación discursiva de la voluntad y la opinión pública, prevaleciendo así el interés general frente al particular. En Habermas, formación ciudadana no sólo subraya la necesidad de la participación ciudadana, sino que aporta a la garantía de los derechos ciudadanos; promueve los ideales de justicia, libertad, desarrollo y autonomía ciudadana; amplia la capacidad de organización y expresión ciudadana; revaloriza la norma; renueva los mecanismos de participación ciudadana y contribuye a la concepción de la formación ciudadana como un medio de formación política, dirigido a la acción eficaz y capaz de generar, a través del ejercicio de la racionalidad comunicativa, tanto cambios mentales como vivenciales (Jiménez Martínez, s.f.: 19)

\section{Conclusiones}

Los distintos aspectos abordados a lo largo del presente ensayo, parten de un diagnóstico de las condiciones actuales de la formación ciudadana en Colombia hasta llegar a una propuesta de formación ciudadana para la Universidad basada en los postulados y principios teóricos de Jürgen Habermas. A partir de lo anterior nos permitimos enunciar las siguientes consideraciones:

1. La formación ciudadana en Colombia continua siendo un concepto al que no se le presta suficiente importancia al interior de la educación. Al desconocer su estrecho vínculo con el paso de la historia; con la transformación permanente de las prácticas y concepciones sobre el ser humano, la educación y la pedagogía; conla noción actual de política y su ampliación a prácticamente la totalidad de los aspectos de la vida social humana y finalmente, con el papel de la sociedad y su responsabilidad junto al Estado, dificulta el ejercicio de prácticas ciudadana. Esto debido a que prescinde de una fundamentación ética, filosófica y política y por endeevidencia una tendencia generalizada a la manipulación velada de la participación política ciudadana de la sociedad colombiana (Jiménez Martínez, s.f.: 8).

2. La consideración de las condiciones presentes en la formación ciudadana en Colombia apuntan a la necesidad de orientar dicha práctica desde la universidad y hacia la comprensión de la educación social actual bajo las perspectivas de los diversos factores políticos que la explican y dan forma. Se hace indispensable que la formación ciudadana en Colombia pase por el análisis, la reflexión, la crítica y la discusión, en un intento por interpretar los cambios históricos colectivos, así como las nuevas realidades sociales que buscan aportar a la construcción de una vida ciudadana y al desarrollo de prácticas 
sociales innovadoras que permitan a la colectividad decidir su presente y disponer por sí misma su futuro.

3. La Universidad en Colombia como escenario social autónomo y privilegiado para la discusión y la reflexión acerca de lo público, debe propender por la pluralidad, la informalidad y la espontaneidad que la asisten. Esto permitirá dirigir la mirada hacia procesos de autoentendimiento ciudadano y al desarrollo de procesos dinámicos de comunicación orientados al logro de acuerdos y consensos.

4. La propuesta de formación ciudadana de Habermas aquí expuesta, subraya la necesidad de incentivar la participación ciudadana en la universidad colombiana, aportando paralelamente a diversos aspectos de la vida social: la garantía de los derechos ciudadanos, la promoción de los ideales de justicia, libertad, desarrollo y autonomía ciudadana, la ampliación de la capacidad de organización y expresión ciudadana, la revalorización de la norma, la renovación de los mecanismos de participación ciudadana y la contribución de una concepción de formación ciudadana como un medio de formación política. Todo lo anterior dirigido a la acción eficaz capaz de generar, a través del ejercicio de la racionalidad comunicativa, cambios tanto mentales como vivenciales (Jiménez Martínez, s.f.: 19).

5. La representación ciudadana no constituye garantía plena ni suficiente para alcanzar los principios razonables de justicia que rigen el modelo de sociedad constitucional (donde los asuntos sustantivos son discutidos y acordados a priori por expertos). Habermas propone como alternativa la posibilidad de llegar a acuerdos mediante el uso racional comunicativo; esto es, a través de la argumentación, la discusión, la autocomprensión y la justicia, que surgen en la esfera pública, toman forma en el discurso y se institucionalizan en una democracia deliberativa, revirtiendo de esta manera el papel pasivo y marginal del ciudadano liberal (Pallas, 2004).

\section{Referencias bibliográficas}

Caballero, J. F. 2006. «La Teoría de la Justicia de John Rawls». Voces y contextos $2(1): 1-22$.

Carvajal Correa, C. 1999. «El concepto de "Autonomía Política" como problema central en el debate entre Habermas - Rawls». Revista de Ciencias Humanas 21: 23-32.

Jiménez Martínez, R. s.f. La Formación Ciudadana en Colombia: una mirada desde la pedagogía social y política. Obtenido el --- en porunaeducaciondecalidad.org/ Congreso/.../Rainiero\%20Jimenez.pdf 
Loos, F. s.f. Habermas, Facticidad y validez. Obtenido el --- en ww.zis-online.com/dat/ artikel/2009_5_318.pdf

Mesa Arango, A. 2008. «La formación ciudadana en Colombia». Uni-Pluri/versidad 8 (3): 1-9.

Ortiz Leroux, S. 2006. «Deliberando sobre la democracia deliberativa. Los dilemas de la deliberación pública». Politica y Sociedad 5 (5): 53-64.

Pallas, C. 2004. «Democracia deliberativa y Formación Ciudadana. Una aproximación a Jürgen Habermas» [Versión electrónica]. Revista Conversación 8:--.

Peralta Duque, B. C. 2007. «La formación ciudadana en el sistema educativo de Colombia: ¿una mirada reactiva o transformadora?» Ponencia VII Encuentro de Grupos y Centros de Investigación Jurídica y Sociojurídica - Medellín.

Torres de Torres, G. M. s.f. Modelos Pedagógicos. Obtenido el -- en http:// gingermariatorres.wordpress.com/modelos-pedagogicos/ 